\title{
Microanalysis of Organic Pigments in Ancient Textiles by Surface-Enhanced Raman Scattering on Agar Gel Matrices
}

\author{
Marilena Ricci, ${ }^{1}$ Cristiana Lofrumento, ${ }^{1}$ Emilio Castellucci, ${ }^{1,2}$ and Maurizio Becucci ${ }^{1,2}$ \\ ${ }^{1}$ Department of Chemistry "Ugo Schiff”, University of Florence, Via della Lastruccia 3-13, 50019 Sesto Fiorentino, Italy \\ ${ }^{2}$ European Laboratory for Non-Linear Spectroscopy (LENS), Via N. Carrara 1, 50019 Sesto Fiorentino, Italy \\ Correspondence should be addressed to Maurizio Becucci; maurizio.becucci@unifi.it
}

Received 31 January 2016; Revised 30 March 2016; Accepted 3 April 2016

Academic Editor: Christoph Krafft

Copyright (C) 2016 Marilena Ricci et al. This is an open access article distributed under the Creative Commons Attribution License, which permits unrestricted use, distribution, and reproduction in any medium, provided the original work is properly cited.

\begin{abstract}
We review some new methods based on surface-enhanced Raman scattering (SERS) for the nondestructive/minimally invasive identification of organic colorants in objects whose value or function precludes sampling, such as historic and archeological textiles, paintings, and drawing. We discuss in detail the methodology we developed for the selective extraction and identification of anthraquinones and indigoids in the typical concentration used in textiles by means of an ecocompatible homogeneous nanostructured agar matrix. The extraction system was modulated according to the chemical properties of the target analyte by choosing appropriate reagents for the extraction and optimizing the extraction time. The system has been found to be extremely stable, easy to use and produce, easy to store, and at the same time able to be analyzed even after long time intervals, maintaining its enhancement properties unaltered, without the detriment of the extracted compound. Highly structured SERS band intensities have been obtained from the extracted dyes adopting laser light excitations at 514.5 and $785 \mathrm{~nm}$ of a micro-Raman setup. This analytical method has been found to be extremely safe for the analyzed substrates, thus being a promising procedure for the selective analysis and detection of molecules at low concentration in the field of artworks conservation.
\end{abstract}

\section{Introduction}

Identification of dyes used in works of art, belonging to different molecular classes, is essential for dating, restoring, and conserving artwork and for studying art history in general. The importance of the cultural artifacts, such as archeological and ancient textiles, drawings, and paintings, requires techniques that can lead to the unambiguous identification of natural dyes from microscopic samples or directly from artworks. The analytical technique that is currently most common for the identification of historical dyes is high-performance liquid chromatography (HPLC) [13]. Although HPLC is a highly specific and very sensitive separation technique, laborious extraction and separation steps could induce molecular changes with a risk of sample loss in separation steps. In addition, its sample requirements (approximately $5 \mathrm{~mm}$ of a thread for textile samples) make it less than ideal for those cases where no or little sampling is allowed. Noninvasive and spectroscopic methods of analysis are usually limited to UV-visible absorption or fluorescence spectroscopy and carried out by means of fiber optics probes and they yield spectra that typically exhibit a poor specificity. Instead, vibrational spectroscopies can provide very specific information on the different materials. However, infrared absorption (or reflectance) is a vibrational spectroscopic technique not ideal for such type of applications because of matrix interference effects. Raman spectroscopy (RS) is another vibrational spectroscopy method. It is based on the observation of weak side-bands in the radiation scattered by the sample upon excitation with monochromatic light. Raman spectroscopy requires no sample preparation and offers the potential of noninvasive analysis, that is, a clear advantage when no sampling is allowed. However, only a limited number of Raman spectra of natural dyes are available in the literature [4-7]; most of the published body of work deals with inorganic pigments [8-11]. The two major factors that have hampered the widespread application of Raman spectroscopy for the identification of natural organic dyes 
are their very high tinting power (i.e., they are likely to be found in works of art in extremely low concentrations) and the strong fluorescence signal generated by excitation with visible light, even when red or near red emitting laser is used and that often obscures the inherently weak Raman scattering signal.

A phenomenon that can significantly enhance the Raman signal intensity is the surface-enhanced Raman scattering (SERS) effect. The Raman scattering efficiency of molecule can be enhanced when the molecule is effectively interacting with a nanostructured metal surface and it is spatially confined within the electromagnetic field of the localized surface plasmon resonance (LSPR) of the nanostructured system $[12,13]$. The localized plasma resonance furnishes a mean to funnel electromagnetic energy in the proximity of the surface of the metallic nanoparticles (NPs), such that light scattering phenomena of species adsorbed on the metallic surface can benefit from a substantial increase in the incoming and outgoing (diffused) electromagnetic fields. SERS effect can give enhancements of Raman signals greater than 8 orders of magnitude. Also, SERS is a selective spectroscopic technique as only molecules effectively interacting with the NPs show enhanced signals. In our specific application on dyed textile fibers, the SERS effect is observed only for the dye molecules and not for the substrate. Finally, it must be mentioned that the presence of metal NPs provides a very efficient way to quench fluorescence from the excited molecules, thus removing a further serious limitation for the application of Raman spectroscopy to dye molecules [12,13]. Therefore, SERS is also very effective for discrimination of molecules of different nature present in artistic samples, and the enhancement of the signal enables us to detect even trace quantities of materials.

Raman experiments can be carried out also at excitation frequencies that are close to (or even resonant) the electronic excitation frequency of the molecule under investigation. In that case, we are dealing with the so-called "resonance Raman" effect that produces much larger signals for specific vibrational modes [14]. Surface-enhanced [resonance] Raman scattering (SE[R]RS) is known to produce further enhancement of the Raman signal. When the LSPR of the enhancing substrate is also in the proper energy region, the SERRS enhancement factor is roughly the product of the enhancement factor for nonresonant SERS and the resonance Raman spectrum intensification factor of the molecule.

In order to improve the quality of the enhancement of the Raman scattering and the reproducibility of the spectra, a wide class of nanostructured substrates such as rough electrodes [15], colloids [16], nanoisland films [17, 18], nanostars [19, 20], nanorods [21], and other nanocomposite technological supports have been carried out. These advancements, together with solid phase microextraction techniques that use synthetic and natural polymeric materials [22], have allowed researchers in the field of cultural heritage to achieve conclusive identification of dyes from artworks with great molecular selectivity and specificity.

The first applications SERS methods for dyes identification on textile fibers were still not enough sensitive to allow nondestructive operations [23]. A first significant step for the increase of sensitivity of this method was represented by the work of Leona [24]. Very small (tens of micrometers) samples containing mordant dyes were treated with HF vapors in order to detach the dye from the mordant. Then, a droplet of colloidal solution containing silver NPs was added to the sample and the dye was free to move and to bind the NPs. Other methods were developed that use a dense dispersion of silver NPs directly on the fibers, leading to an irreversible contamination of the original sample $[25,26]$. An innovative and effective method for dyes microextraction followed by SERS dyes identification was recently presented [27]. It was based on the use of common hydrogels based on hydroxymethacrylates (contact lens blanks), loaded with water, organic solvents, and chelating agents as extraction media. Another method was presented based on the application to the sample surface of a cellulose film loaded with silver NPs [28]. The film dries in minutes after application and SERS spectra of anthraquinonic dyes present on the surface were obtained with good sensitivity. The dimensional changes associated with the drying process possibly induces mechanical stress on the sample surface leading to the detachment of small fragments. The film self-detaches or is peeled off by the operator; its formulation was optimized in order to minimize the gel penetration in the samples, to obtain possibly its complete detachment, and to limit the detachment of small particles from the sample surface [29]. Comprehensive reviews on the application of SERS to forensic science and cultural heritage have been already published [30, 31].

This review will discuss the nondestructive microextraction procedure we developed for dye extraction from textile fibers, based on the use of an environmentally friendly nanocomposite hydrogel, to provide a new tool for those researchers who would like to embark on this kind of work using the SERS technique. We will present the results obtained on mordant dyes (anthraquinones) and vat dyes (indigoids) used both in mockups prepared in the laboratory and in ancient textiles.

\section{Materials and Methods}

2.1. Dyeing of Textiles. Dyes can be divided into three different categories (mordant, vat, and direct dyes), depending on the procedure according to which they are applied to fabrics. In what follows, we are providing information of the different classes of natural organic colorants used in textile dyeing in order to clearly outline the approach we are using.

Colorants named "mordant dyes" do not have a strong chemical affinity for the textile fibers, which therefore require being treated prior to the dyeing stage, involving a two-step chemical reaction. A solution of a "mordant" is first used to impregnate the fibers, allowing the metal ion to become complexed to appropriate functional groups in the structure of the textile. During the dyeing process, the colorant interacts with the mordant-fiber complex via ionic and coordinate covalent bonds to form insoluble brightly colored species. The complex thus formed within the fibers does not easily wash out of the textile and the resulting dyeing is therefore relatively fast. The mordant helped the dye bite onto the 
fiber so that it would hold fast during washing. A scheme of the binding mechanism for alizarin on cotton fibers is shown in Figure 1. Aluminum, iron, tin, chromium, or copper ions, as well as tannins, are examples of mordants; one of the substances most commonly employed in ancient times for this purpose was potassium alum $\left(\mathrm{KAl}\left(\mathrm{SO}_{4}\right)_{2} \cdot 12 \mathrm{H}_{2} \mathrm{O}\right)$, but iron sulfate $\left(\mathrm{FeSO}_{4} \cdot 7 \mathrm{H}_{2} \mathrm{O}\right)$ and tin chloride $\left(\mathrm{SnCl}_{2}\right)$ were often used as well. Commonly employed in association with mordants are dye-assistants, such as cream of tartar or oxalic acid, which brighten the colors, protect the fibers, and help the absorption of mordants. Mordant dyes can be used with wool, silk, and protein yarns while cellulose fibers such as linen and cotton have to be chemically modified before dyeing. The vast majority of natural colorants belongs to this category and yields different colors when combined with different mordants: a typical example is represented by the madder lake, which can produce red, orange, or violet shades when associated with aluminum, tin, and iron mordants, respectively.

Colorants belonging to the "vat dyes" class are waterinsoluble but, under reducing conditions, they can be converted into a leuco form, soluble in alkali. Nowadays the reducing reagent used is sodium dithionite, $\mathrm{Na}_{2} \mathrm{~S}_{2} \mathrm{O}_{4}$. Immersion of the textile into the dye solution allows the dissolved molecules of colorant to penetrate the fibers. After that, upon removal of the wet dyeing from the bath and exposure to atmospheric oxygen, these substances can be oxidized back to their colored forms which, thanks to their insolubility in water, are trapped on the surface of the fiber. The well-known indigo and woad blue colorants, as well as Tyrian purple, belong to this category. The relevant chemical reactions involved in the dyeing process with indigo are outlined in Figure 2.

Even if direct dyes are applied directly to the fiber without any special treatment, usually they are less wash- and lightfast than vat or mordant dyes. Examples of direct dyes include turmeric and saffron, which can be fixed to all fibrous materials in aqueous solution.

2.1.1. Mockups Dyed with Anthraquinones. Three pieces of cotton fabric were prepared according to traditional dyeing methodologies, by using alizarin (Sigma-Aldrich), purpurin (Sigma-Aldrich), and carminic acid (Sigma-Aldrich). The textiles have been treated prior to the dyeing step with an alum (Zecchi, Firenze) mordant solution in distilled water (J. T. Baker HPLC Gradient Grade). After the dyeing step, they have been thoroughly washed with distilled water a couple of times and allowed to dry.

2.1.2. Mockups Dyed with Indigoids. Pieces of cotton have been dyed according to the recipe reported by Schweppe [32]. The dyeing liquor was prepared by stirring $15 \mathrm{~g}$ of indigo powder with $75 \mathrm{~mL}$ of warm water in a beaker glass until it formed a paste. In a second vessel $30 \mathrm{~g}$ of sodium hydroxide, $\mathrm{NaOH}$, was dissolved in $120 \mathrm{~mL}$ of warm water. A portion of this solution $(60-70 \mathrm{~mL})$ was poured over the indigo paste, stirring vigorously. Then, $30 \mathrm{~g}$ of sodium dithionite, $\mathrm{Na}_{2} \mathrm{~S}_{2} \mathrm{O}_{4}$, was added while keeping stirring and
$1 \mathrm{~L}$ of warm water was added while stirring carefully. This mixture was heated to $55^{\circ} \mathrm{C}$. Clean pieces of cotton were immersed in warm water until the fabric was thoroughly wet. The fabric was then put inside the dyeing liquor and kept in the dye-bath for some minutes. The pieces of cotton were then taken out of the vat squeezing the liquor out thoroughly. When the fabric comes out of the vat it has a typical greenyellow color, which turns blue when exposed to air. A special precaution was used with the Tyrian purple indigoid dye. The dyeing procedure adopted was similar to the previous one for indigo, using a colorant $/ \mathrm{NaOH} / \mathrm{Na}_{2} \mathrm{~S}_{2} \mathrm{O}_{4}$ ratio equal to $1: 2: 4$. Since Tyrian purple is a mixture of brominated indigoids, 6-bromoindigo (MBI) and 6,6' dibromoindigo (DBI), it is subject to debromination because of the action of UV-light. For this reason, when the reagents were heated to $55^{\circ} \mathrm{C}$, the light was turned off and the flask was wrapped in aluminum foil. A yellow-greenish homogeneous solution was obtained and the wet fabric was put into the dyeing bath for 15 minutes. Then it was removed from the flask and exposed to air while still protected from light. After one hour of air exposure, the fabric was rinsed in aqueous solution and allowed to dry.

2.2. Silver Nanoparticles for SERS. We used the standard chemical synthesis of Ag nanoparticles (AgNPs) devised by Lee and Meisel [16]. In order to improve the production of suitable nanoparticles (NPs) with an average diameter of $40 \mathrm{~nm}$, we took care of placing the reaction flask, after the reduction step, in an ice bath [33]. The average diameter of the particles was checked from their UV-Vis absorption spectrum, which showed a $100 \mathrm{~nm}$ broad peak centered at $418 \mathrm{~nm}$, in good agreement with previous reports [16].

2.3. Agar Gel. The agar gel usage as the extraction medium was suggested by its intrinsic safety for the operator and its widespread use for the cleaning of artistic materials [34]. It was prepared from agar-agar, a commonly gelling material used for food preparation. Agar-agar is a polymer containing mostly $D$-galactose units. Agar-agar was repeatedly washed in order to remove possible contaminants and chloride ions. The gel can be easily prepared by mixing $1 \mathrm{~g}$ of agaragar with $50 \mathrm{~mL}$ of water, warming it for few seconds in a common microwave oven ( $300 \mathrm{~W}$ power) and cooling down the viscous solution in a convenient glassware (Petri dish). The agar gel becomes rapidly rigid and stable at room temperature. It is important to notice that the agar gel is a very good substrate for bacteria cultures so its practical lifetime could be very short and SERS experimental results can be altered by the presence of such kind of contamination. A convenient practice resulted to be using the colloidal solution containing AgNPs in place of water for the agar gel preparation. It provides a double advantage. On one side the AgNPs act as a bacteriostatic agent, thus preventing the agar gel biological contamination: we have found that this kind of material (Ag-agar), stored in closed containers, can be maintained at room temperatures in the dark for a long time (a few months). On the other side, the agar gel used as dyes extraction medium already carries the AgNPs needed 


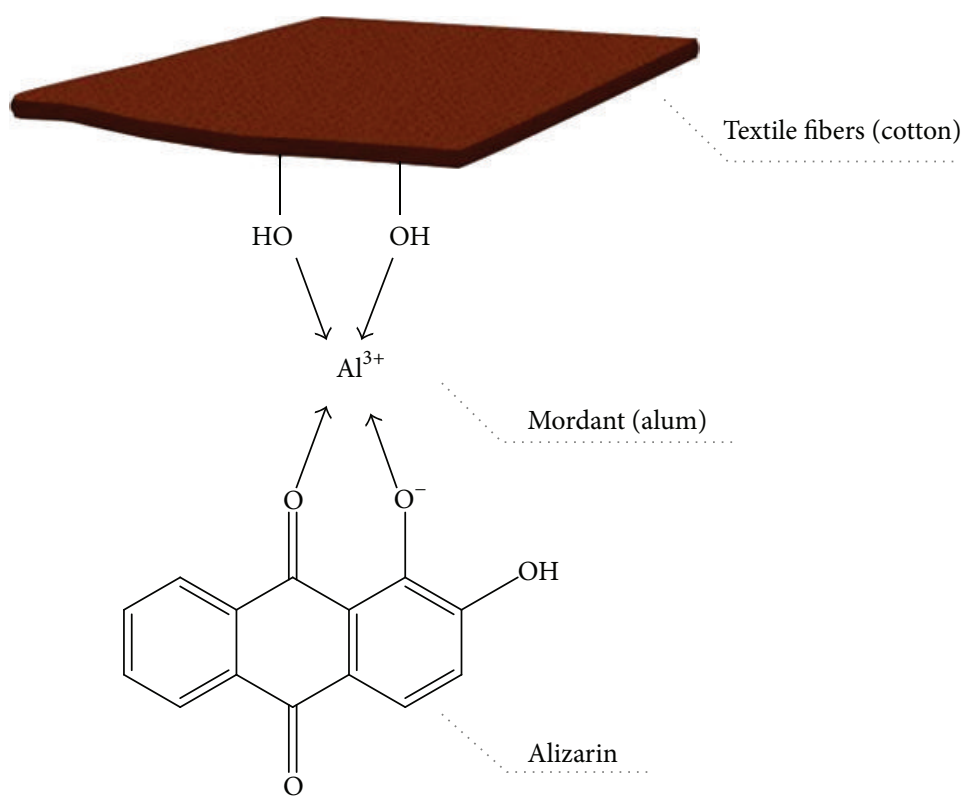

FIGURE 1: Chemical structure of alizarin anion and the bonding mechanism to the cotton fiber mediated by the presence of the $\mathrm{Al}^{3+}$ ion from the mordant.

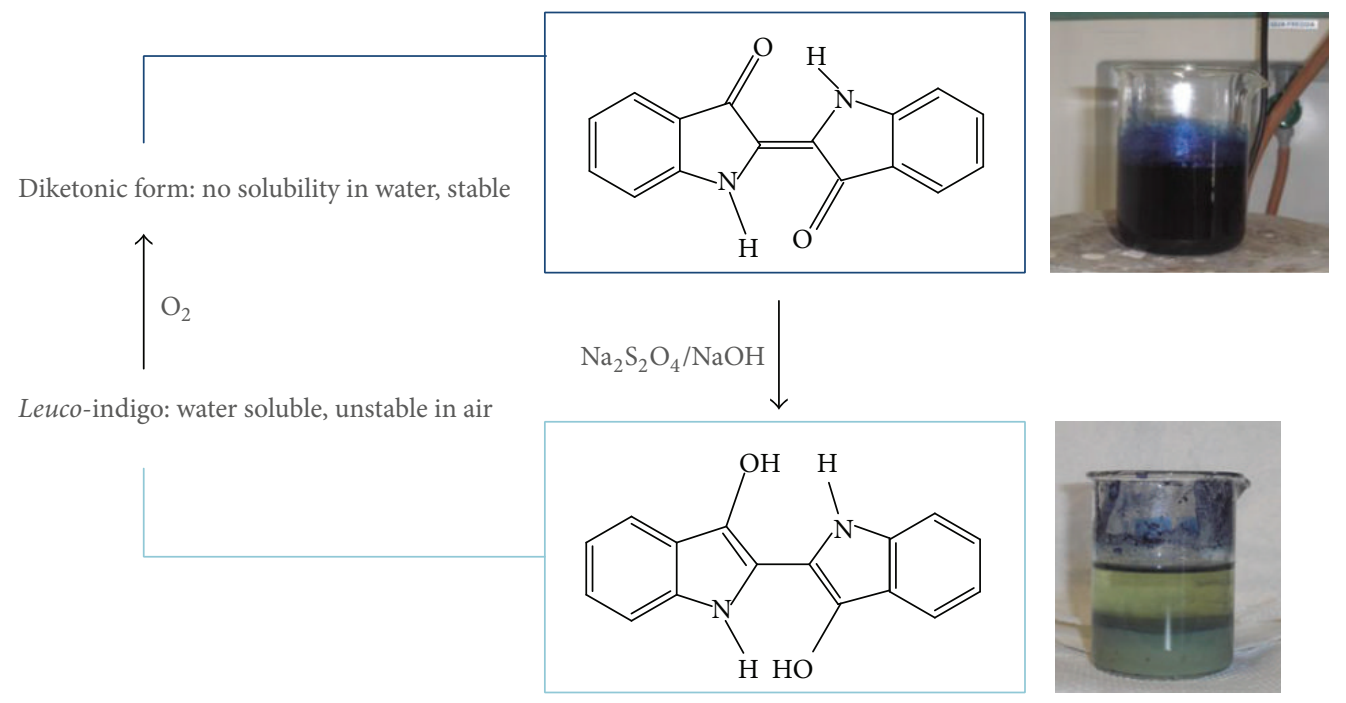

FIgURE 2: Chemical reactions involving indigo during the dyeing process. The indigo blue dye is the diketonic form.

for the SERS measurements. Anyway, droplets of the AgNPs colloidal solution can be poured in the top of the agar gel beads we use, after the extraction process, in order to further improve SERS measurements.

2.4. Sample Extraction. The agar gel can act as a suitable extraction substrate for dyes on textile fibers. Clearly, water already present in the agar gel is not, per se, a suitable solvent for the dyes extraction. Different extraction strategies can be used for different dyes classes. Here we are reporting examples of studies on anthraquinonic $[35,36]$ and indigoid dyes [37].

Anthraquinonic dyes, like alizarin and carminic acid, are bound to textile fibers by the use of a mordant like alum that introduces $\mathrm{Al}^{3+}$ ions. These ions act as a bridge between the fiber and the dye molecule (Figure 1). Both molecules can be tentatively transferred from the textile fiber to the agar gel either by using a solvent in which they are highly soluble (e.g., an alcohol) or by detaching them from the fiber by specific chemical processes. Therefore, in the first approach we added a few ethyl alcohol droplets to the Ag-agar and then we place 
it in contact with the fiber for a suitable amount of time (the extraction time was 30 minutes). In the second approach, we tried to detach only the dye from the fiber. Even though the chemical etching with HF method was already well established [23], we did try to avoid it as it requires the use of highly toxic chemicals in sealed chamber and the removal of fibers from the original artifact. We used a polydentate chelating agent, ethylenediaminetetraacetic (EDTA) acid, as the reactant is able to detach the dye by selective removal of the $\mathrm{Al}^{3+}$ ions from the system. We added $0.5 \mathrm{~g}$ of EDTA (or equivalent amount of its disodium salt) to the mixture described above, during the agar gel preparation. The use of EDTA leads to an acidic system $(\mathrm{pH} \approx 3)$ while the use of its disodium salt leads to solutions at $\mathrm{pH}$ values almost neutral. A bead (a small cube of $5 \mathrm{~mm}$ side) of Ag-agar containing also EDTA was then placed in contact with the textile for some time (the extraction time ranges from 15 to 30-50 minutes depending on the textiles being analyzed).

Indigo and related dyes (like Tyrian purple) are waterinsoluble dyes that are dissolved in the textile fibers in their reduced leuco form. Once dispersed within the fiber, the natural oxidation process, due to the exposure of atmospheric oxygen, reverts the dye to its colored form which then sticks to the fiber. We added to the Ag-agar gel the chemical reactants used for the chemical reduction of the indigoid dye. Therefore, during the contact of the bead, we tried to convert indigo into its leuco form, which is water soluble, thus possibly transferable to the agar gel. Specifically, we added to the bead a droplet of the $\mathrm{NaOH} / \mathrm{Na}_{2} \mathrm{~S}_{2} \mathrm{O}_{4} 1: 2 \mathrm{w} / \mathrm{w}$ water solution described above. The bead was then applied to the textile fibers, covered with a glass to reduce evaporation, and let in contact for 5-15 minutes

In both cases, after the chosen contact time with the fiber, the bead (still wet) was removed from the textile and let dry in air. In about $30^{\prime}$ the bead loses most of its water content (we measure a loss of about $92 \%$ in weight) and reduces considerably its dimensions (from a $5 \mathrm{~mm}$ size cube it goes down to a 1 or $2 \mathrm{~mm}$ side film, about $0.2 \mathrm{~mm}$ thick). The dried bead was then moved to the Raman microscope for the measurement of the SERS spectrum.

2.5. SERS Measurements. We used a micro-Raman spectrometer RM2000 from Renishaw operating with laser excitation either at 514 or $785 \mathrm{~nm}$ and equipped with a CCD detector thermoelectrically cooled. It operates in backscattering geometry and uses a 50x microscope objective from Leica. The typical dimension of the active area of the sample is about $2 \mu \mathrm{m}$ and the used laser power is in the order of $20-200 \mu \mathrm{W}$ at the sample.

\section{Results and Discussion}

The Ag-agar gel shows an open, and porous structure with channels of 50-200 nm diameter. The AgNPs can be found in the support either as isolated particles or as aggregates; their sizes range between 50 and $450 \mathrm{~nm}$, in accordance with the UV-Vis absorption spectrum. A high-resolution image of this nanostructured material (Figure 3) was obtained by

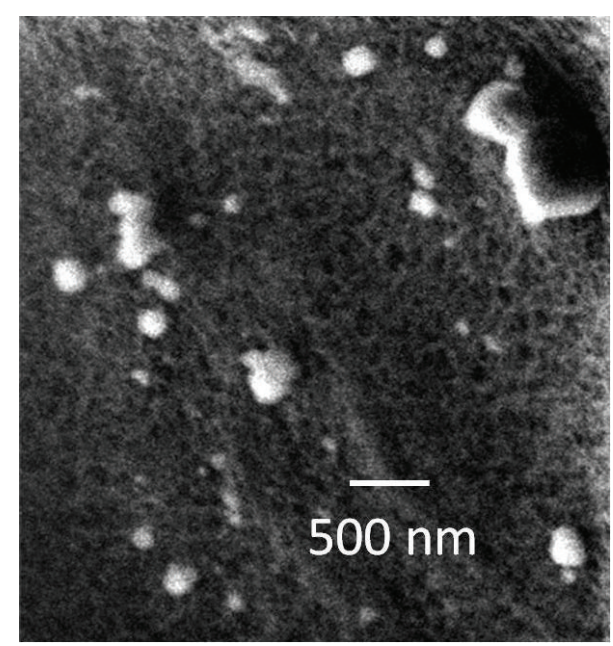

FIGURE 3: Helium ion microscope image of the native agar gel loaded with Ag nanoparticles.

using a Helium ion scattering apparatus (Orion Plus by Carl Zeiss), a system well suitable for the characterization of soft materials without any preliminary metallization treatment. The experimental details for sample preparation and the measurement settings are described in our previous report [37].

Upon a convenient contact time with the fibers, the Ag-agar bead was removed from the sample and allowed to dry in air, thus decreasing in volume. It is a factor that positively affects the Raman signal as it corresponds to an effective sample concentration process and also by decreasing the distance between the metal NPs, it favors the presence of stronger local electric fields [13]. Also, if the dehydration process is not completed the dimension of the bead is not constant and the focusing on the sample in the microscope is troublesome. As a final remark, all our tests demonstrated that the agar gel can be conveniently used as SERS substrate for microextraction as it shows an intrinsic negligible Raman/SERS signal that makes the measurements practically background-free.

The first tests for our microextraction/concentration/ SERS determination approach were carried out on the mockups prepared with the alizarin dye [35]. These tests were further extended to include different kinds of fibers and substrates [36]. The preliminary tests were mostly oriented to verify the extraction efficiency and the safety with respect to the textile fiber. We found that both the use of ethanol and EDTA solution as an extraction-promoting reagent on the Ag-agar beads made it possible to observe the SERS signal from alizarin already after 10-minute extraction time. In difficult cases, the extraction time can be as long as 50 minutes. In both cases, during extraction, the bead must be covered in order to prevent fast evaporation of the solvents.

We report in Figure 4 the SERS spectra obtained from the dried beads after extraction of the red dye from a pre-Columbian textile sample with Ag-agar gel spiked with either ethanol or disodium-EDTA solution. Both spectra correspond to the well-known Raman spectrum of the 


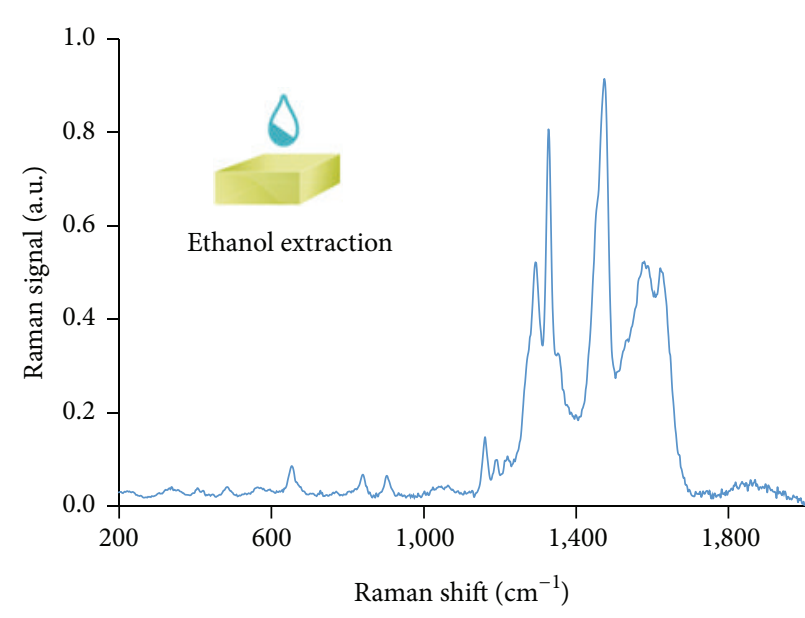

(a)

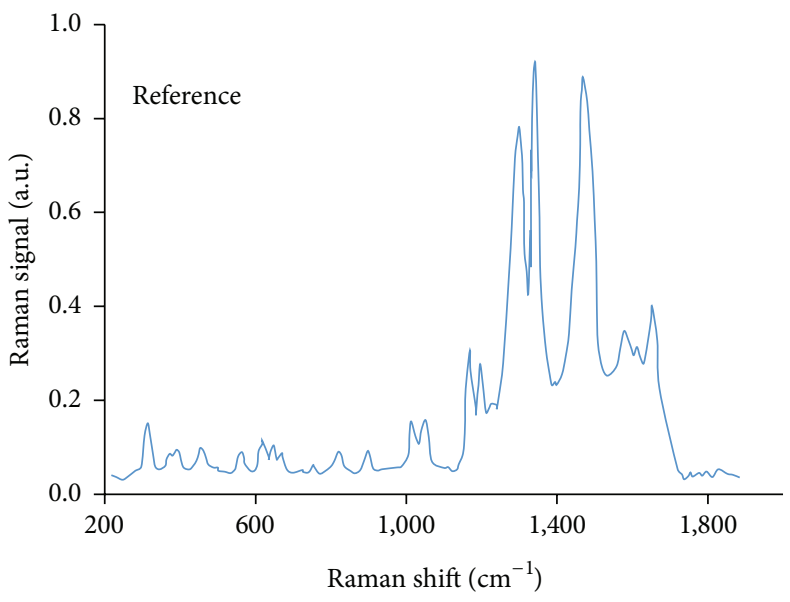

(c)

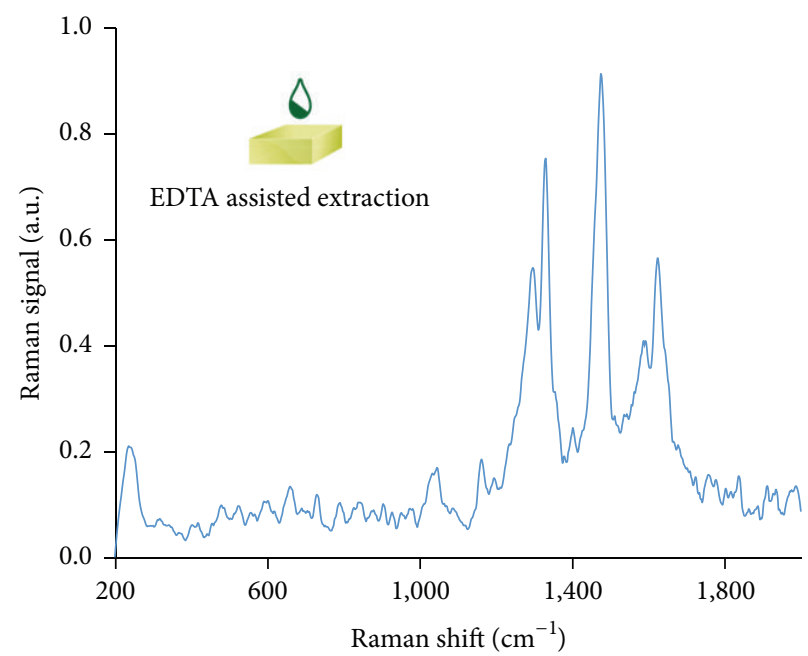

(b)

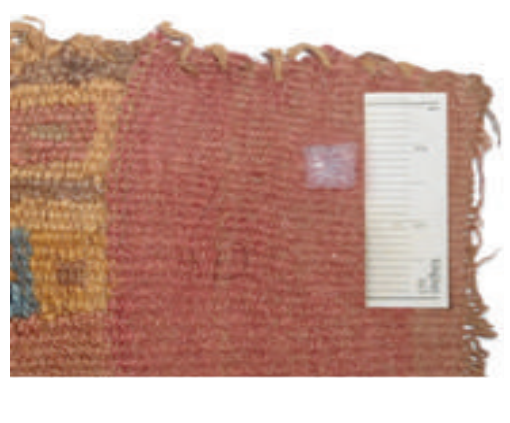

(d)

FIGURE 4: SERS spectra (514 nm excitation) obtained from the dried beads after extraction of the red dye from a pre-Columbian textile sample with Ag-agar gel spiked either with ethanol or disodium-EDTA solution ((a) and (b), resp.) or from reference alizarin solutions in ethanol (c). (d) The textile sample is shown with a $25 \mathrm{~mm}$ ruler on top; the $5 \mathrm{~mm}$ dimension agar gel bead is placed close to the ruler.

monoanionic species of the alizarin dye [38]. However some attention is needed when the experimental data are evaluated. Alizarin is an acid and it is well known that different species exist in solution at different $\mathrm{pH}$ values. Alizarin exists as a neutral molecule in acidic solutions while in neutral solutions the most abundant species is the monoanionic one while at $\mathrm{pH}$ above 10 the dianionic species is dominant. Alizarin is also an acidochromic molecule that changes its color with $\mathrm{pH}$ : the neutral molecule is red, the monoanion is yellow and the dianion is blue. Thus, while performing Raman experiments on alizarin two key factors must be taken into account [38]. The first is the $\mathrm{pH}$ as it controls the effective species present in solution, the second is the excitation wavelength used for the Raman experiment. The chemical nature of the dye changes with $\mathrm{pH}$; then both its color and the possible resonance Raman effects change as well. All of these phenomena will reflect directly on the shape and intensity of the different vibrational bands observed in the Raman spectrum. Therefore, a very good knowledge of the chemical system is needed and a clear feeling of the relevance of the different experimental parameters is also mandatory. In this context, the prediction of Raman spectra of alizarin and other dyes by density functional theory has provided a systematic information on these systems and assisted the interpretation of the experimental data collected by SERS $[38,39]$.

Additional tests were carried out concerning the alizarin extraction from textile substrates made with different fibers: wood and silk. We added the EDTA reagent to the Agagar bead for both the considered textile substrates, and we left the agar beads in contact with the fibers for $50 \mathrm{~min}$ and $15 \mathrm{~min}$, respectively. We succeeded in acquiring excellent SERS spectra of alizarin in both cases, thus confirming the capability of the procedure to extract alizarin from different textile fibers [36].

The safety of the process with respect to the textile fiber was verified by the determination of the color of the fiber in the sampling area before and after the contact with the Ag-agar bead. Colorimetric measurements on the textile 


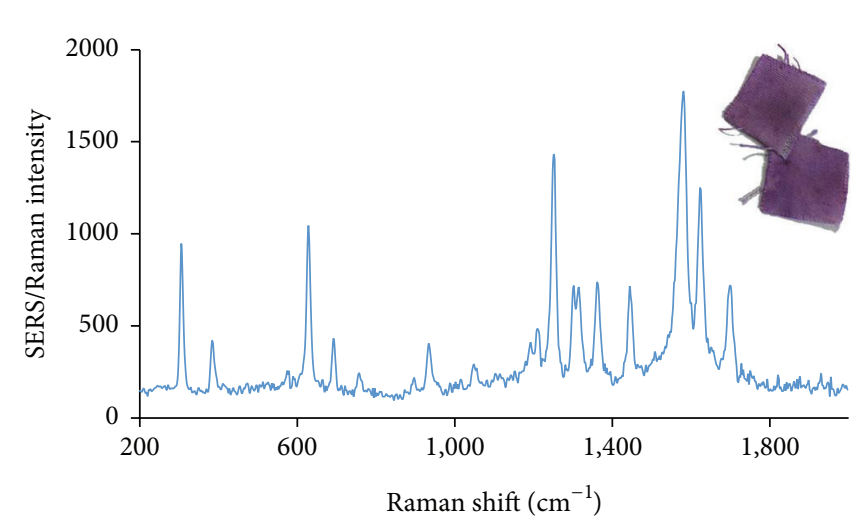

(a)

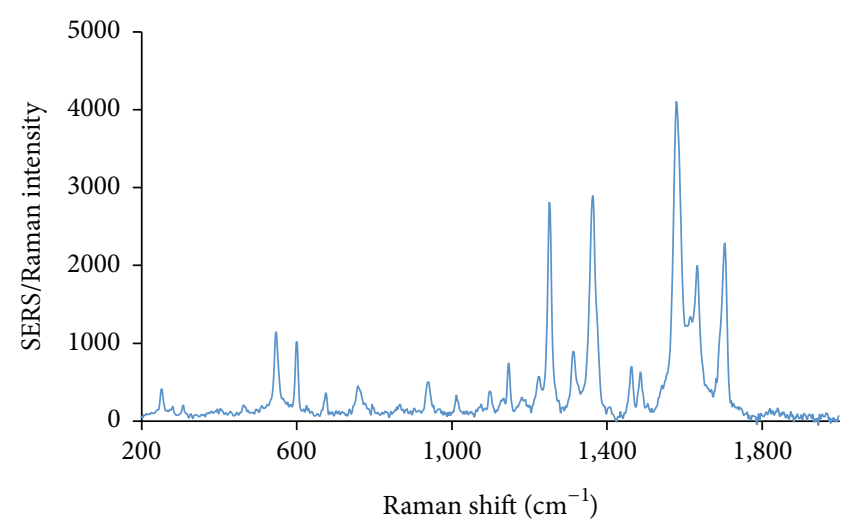

(b)

FIGURE 5: SERS spectrum (514 nm excitation) of Tyrian purple extracted from a cotton fiber mockup by using an agar gel bead containing the leuco-reduction chemicals (a) and Raman spectrum indigo crystals (b).

fiber were made by using a CM-2600d Konica-Minolta portable spectrophotometer equipped with the integrative sphere and a Xenon lamp. The measurement aperture is $3 \mathrm{~mm}$ diameter; the light that reaches the detector is reflected by the illuminated surface with an angle of $8^{\circ}$. Color coordinates are based on CIE $L^{*} a^{*} b^{*}$ system using an illuminant D65 with an observer angle of $10^{\circ}$. The value of $\Delta E^{*}=2.3$ corresponds to a just noticeable difference in color [40]. The results reported in Table 1 demonstrate that the fibers are virtually nonaltered by our extraction process.

After extraction, traces of the chemical components of the bead were searched on the textile surface by spectroscopic methods (ATR-FTIR and X-ray fluorescence methods). X-ray fluorescence analysis was performed with a Bruker TRACeR III-SD spectrometer and the spectra were acquired in 50 seconds at $40 \mathrm{KV} / 15 \mu \mathrm{A}$ on the rhodium X-ray tube. No evidence for the presence of silver on the fiber was detected. ATR-FTIR did not reveal any surface contamination from the agar gel.

The first test for extraction of indigoids by water solubilization of the dye in its reduced leuco form was carried out on a mockup textile purposely prepared of Tyrian purple [37]. The test is important in order to assess the extraction efficiency and the invasiveness of the process with respect to the textile sample. Even though the chemical reagents involved are quite strong (water solution of $\mathrm{NaOH}$ and $\mathrm{Na}_{2} \mathrm{~S}_{2} \mathrm{O}_{4}$ ) we consider them rather safe for the textile fiber as they are used also in the original dyeing process. Also, they are applied only in very small amounts and in a limited area. Finally, in the case of relevant contamination, being water soluble, they can be washed out easily.

The efficiency of the extraction process was measured by looking at the SERS spectrum that was possibly obtained from the dye extracted on the bead. The SERS spectrum obtained from the dye extracted on the bead and measured on the dried bead with the micro-Raman spectrometer upon $514 \mathrm{~nm}$ excitation is reported in Figure 5. The excitation conditions we used do not allow for the best sensitivity in the indigo detection by Raman measurements as it is well known that electronic resonance effects strongly enhance the indigo Raman signal when a red excitation is used [41]. However, a very good signal to noise ratio is obtained in the observed spectrum. It is quite relevant to appreciate that the extraction process does not lead to the decomposition of the Tyrian purple (6,6 dibromoindigotin) dye. It is well known that this dye can decompose by the breaking of the $\mathrm{C}-\mathrm{Br}$ bonds. The bands corresponding to the $\mathrm{C}-\mathrm{Br}$ stretching mode at $305 \mathrm{~cm}^{-1}$ are clearly observed in the SERS spectrum.

The method was then applied to a small piece of blue wire in wool, silk, silver, and gilded silver obtained from the Medici's XVIth century tapestry (Joseph escaping from Potiphar's wife, designed by Bronzino). The Ag-agar bead containing the leuco-reagents was applied for 10 minutes to the fiber. No sensible discoloration was appreciated on the fiber after the bead removal. The SERS spectrum obtained from the dried bead is reported in Figure 6 and it clearly shows the characteristic pattern of bands associated with indigo (shown in Figure 5(b)).

The same tests for the safety of the extraction procedure described above were repeated also in the case of this series of measurements. As reported in Table 2, the changes of chromatic properties of the mockup textile fiber upon extraction are negligible, even for extraction times much longer than those needed for obtaining a sample for SERS measurements (10 minutes).

$\mathrm{X}$-ray fluorescence analysis revealed a single detectable signal upon application of the extraction bead. It was related to Sulphur and it was observed only after $30^{\prime}$ contact time. ATR-FTIR did not show any evidence of surface contamination.

Finally, we verified if the methods we propose were specific for the different dyes. It is quite clear that extraction promoted by the presence of a solvent, for example, ethanol, is not specific. All the dyes that are not water soluble but have some affinity with organic solvents can be extracted, even if with different efficiency. We did try to use the EDTA method developed for mordant dye with the mockup dyed with indigo and no SERS signal from indigo was detectable. The use of the 


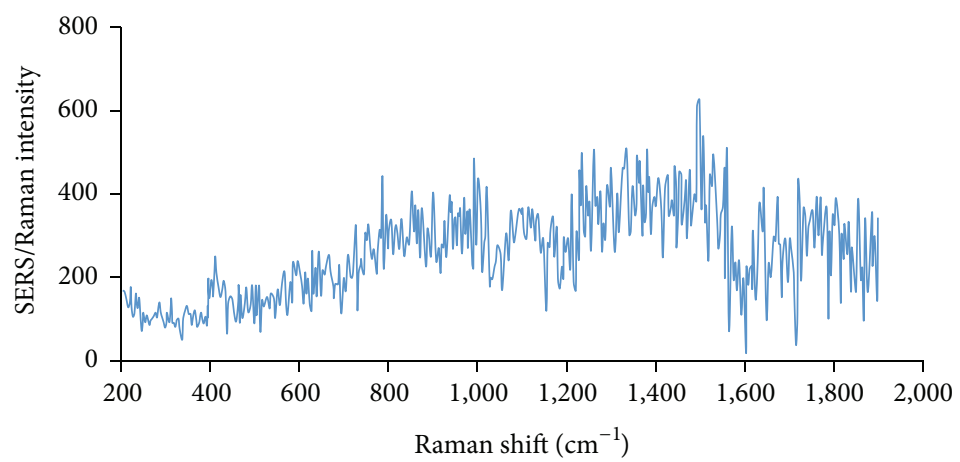

(a)
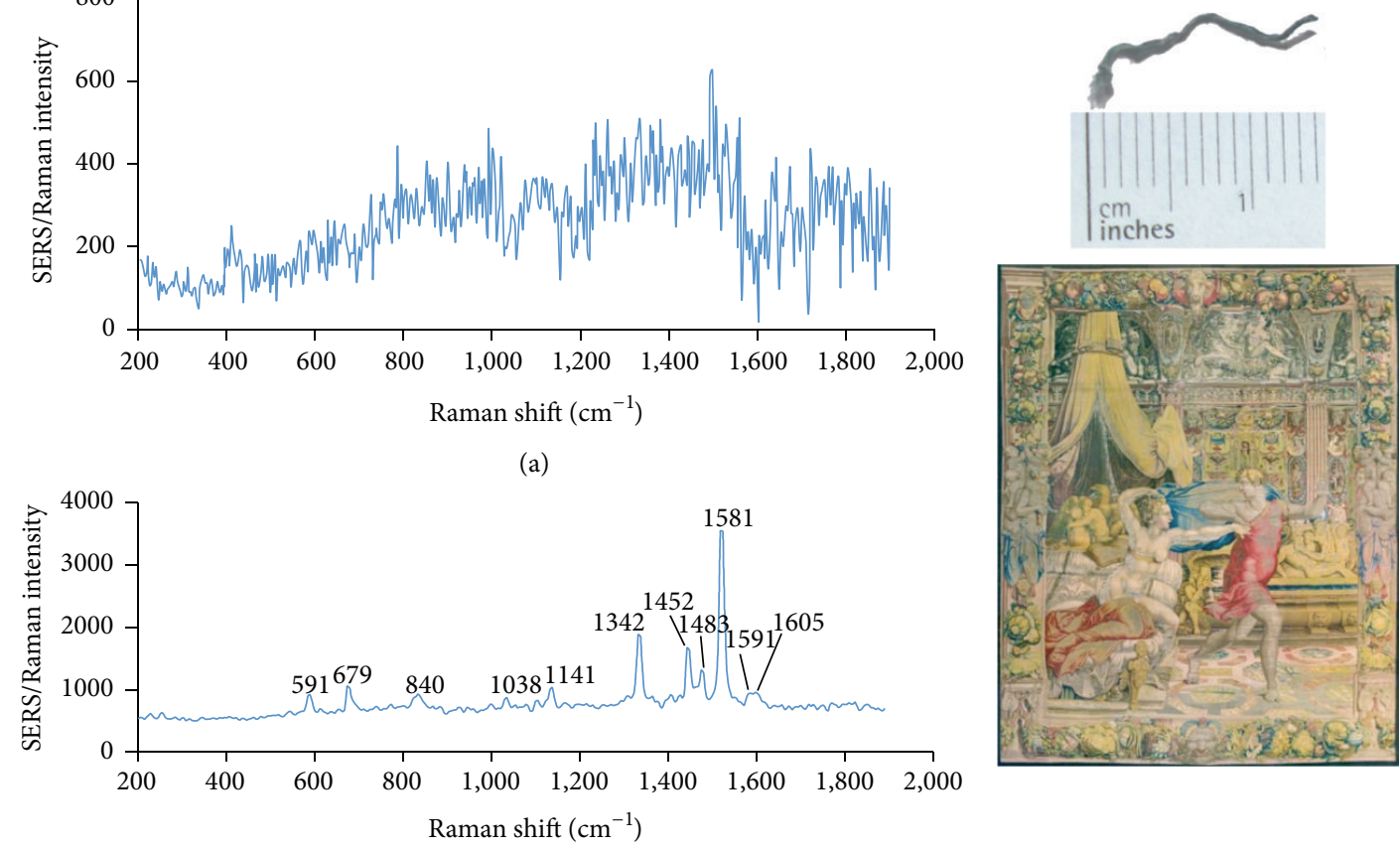

(c)

(b)

FIGURE 6: Upper trace: Raman spectrum from the blue thread of an XVIth century tapestry (Italian artwork) shown in (b). Lower trace: SERS spectrum (514 nm excitation) of indigo extracted from the same sample by using an agar gel bead containing the leuco-reduction chemicals. Adapted from [37].

TABLE 1: Change in color (CIE $L^{*} a^{*} b^{*}$ system) of the cotton mockup dyed with alizarin upon extraction with the Ag-agar gel added with either ethanol or disodium-EDTA solution.

\begin{tabular}{|c|c|c|c|c|c|c|c|}
\hline & \multicolumn{3}{|c|}{ Before } & \multicolumn{4}{|c|}{ After } \\
\hline & $L^{*}$ & $a^{*}$ & $b^{*}$ & $\Delta L^{*}$ & $\Delta a^{*}$ & $\Delta b^{*}$ & $\Delta E^{*}$ \\
\hline $\begin{array}{l}\text { Ethanol } \\
15 \text { minutes }\end{array}$ & 44.39 & 45.73 & 21.02 & 0.06 & -0.04 & -0.03 & 0.08 \\
\hline $\begin{array}{l}\text { Disodium-EDTA } \\
30 \text { minutes }\end{array}$ & 45.08 & 45.9 & 20.8 & 0.08 & -0.03 & -0.04 & 0.09 \\
\hline
\end{tabular}

TABLE 2: Change in color (CIE $L^{*} a^{*} b^{*}$ system) of the cotton mockup dyed with indigo upon extraction with the Ag-agar gel added with the leuco-reagents.

\begin{tabular}{lccccrr}
\hline & \multicolumn{3}{c}{ Before } & & & After \\
& $L^{*}$ & $a^{*}$ & $b^{*}$ & $\Delta L^{*}$ & $\Delta a^{*}$ & $\Delta b^{*}$ \\
\hline $\begin{array}{l}\text { Point 1: } \\
60^{\prime} \text { contact time }\end{array}$ & 36.36 & -1.59 & -22.51 & 0.00 & 0.11 & -0.42 \\
\hline $\begin{array}{l}\text { Point 2: } \\
120^{\prime} \text { contact time }\end{array}$ & 37.29 & -1.77 & -22.30 & -0.16 & -0.03 & 0.43 \\
\hline
\end{tabular}

leuco-reagents on the alizarin mockup have made the fiber to change temporarily color from red to blue (the high $\mathrm{pH}$ value of the leuco-reagents solution induced deprotonation of alizarin) but no extraction of the dye was observed.

\section{Conclusions}

The paper summarizes the development of a SERS substrate (synthesized by mixing a hydrogel, agar-agar, with a colloidal dispersion of silver nanoparticles) used to extract minimal amounts of dye molecules from textiles. The intent is to provide a simple, efficient, and safe method to extract colorants from textiles fibers of historical, artistic, and archeological interest, exploiting then the high sensitivity of SERS spectroscopy for their identification. The method was found to be very efficient and has advantages with respect to other possible methodologies [23-29]. It is a nondestructive method that is possibly applied directly on the original 
fibers, without sampling. The safety of the methodology was assessed by means of X-ray fluorescence, ATR-FTIR, and colorimetric analyses that confirmed that no residuals or discoloring effects have been observed on the textile surface after the microextraction, once the extraction process has been optimized with tests on mockups. The simplicity of the procedure to perform SERS measurements, the stability of the dry nanocomposite gel, and the strong enhancement of the observed SERS signal are factors that assure the detection and the recognition of dyes. The high level of enhancement achieved is due also to the shrinkage of Ag-agar gel structure upon drying, which concentrates the extracted molecules and possibly favors the interaction of silver nanoparticles that create high plasmon density sites. Moreover, using a gel as a medium for the solvent mixture allows for a more localized and controlled microextraction essay, which involves the analysis of extremely small areas of the object under study.

Furthermore, the possibility of selecting different solvents and components within the gel structure for the improvement of the extractive performance of the nanocomposite matrix offers the possibility of tailoring the gel for the extraction of specific molecules and for the potential discrimination and recognition of single components of a mixture.

In particular, the efficacy of the proposed methodology has been widely demonstrated by its application to several textiles of artistic relevance, validating the suitability of the proposed method on the investigated samples. The high efficiency of this methodology confirms that the tailored gel extraction is a promising nondestructive technique for SERS identification of dyes.

Further studies are under way considering other substrates for extraction, in order to extend the application of the method to other classes of materials of artistic and historical interest, such as illuminated manuscripts, paintings, and sculptures. As a possible development of this method, we foresee its integration with current cleaning methods based on the use of agar gel. This would allow for simultaneous cleaning and sampling actions to be followed by instrumental characterization of the removed materials.

\section{Competing Interests}

The authors declare that there are no competing interests regarding the publication of this paper.

\section{Acknowledgments}

This work was supported by Italian MIUR (PRIN, Grant 2010329WPF_007) and by Ente Cassa di Risparmio di Firenze (Grant no. 2014.0405A2202.8044). Opificio delle Pietre Dure (Florence, I) and the Museo del Tessuto (Prato, I) made available samples of ancient textile artworks for our research. Their support is kindly acknowledged.

\section{References}

[1] I. Degano, E. Ribechini, F. Modugno, and M. P. Colombini, "Analytical methods for the characterization of organic dyes in artworks and in historical textiles," Applied Spectroscopy Reviews, vol. 44, no. 5, pp. 363-410, 2009.

[2] M. R. van Bommel, I. V. Berghe, A. M. Wallert, R. Boitelle, and J. Wouters, "High-performance liquid chromatography and nondestructive three-dimensional fluorescence analysis of early synthetic dyes," Journal of Chromatography A, vol. 1157, no. 1-2, pp. 260-272, 2007.

[3] Z. C. Koren, "HPLC analysis of the natural scale insect madder and indigoid dyes," Journal of the Society of Dyers \& Colourists, vol. 110, no. 9, pp. 273-277, 1994.

[4] L. Burgio and R. J. H. Clark, "Library of FT-raman spectra of pigments, minerals, pigment media and varnishes," Spectrochimica Acta Part A: Molecular and Biomolecular Spectroscopy, vol. 57, no. 7, pp. 1491-1521, 2001.

[5] F. Casadio, M. Leona, J. R. Lombardi, and R. Van Duyne, "Identification of organic colorants in fibers, paints, and glazes by surface enhanced Raman spectroscopy," Accounts of Chemical Research, vol. 43, no. 6, pp. 782-791, 2010.

[6] S. Bruni, V. Guglielmi, and F. Pozzi, "Historical organic dyes: a surface-enhanced Raman scattering (SERS) spectral database on Ag Lee-Meisel colloids aggregated by $\mathrm{NaClO}_{4}$," Journal of Raman Spectroscopy, vol. 42, no. 6, pp. 1267-1281, 2011.

[7] P. Colomban and D. Mancini, "Lacquerware pigment identification with fixed and mobile raman microspectrometers: a potential technique to differentiate original/fake artworks," Arts, vol. 2, no. 3, pp. 111-123, 2013.

[8] A. Zoppi, C. Lofrumento, M. Ricci, E. Cantisani, T. Fratini, and E. M. Castellucci, "A novel piece of Minoan art in Italy: the first spectroscopic study of the wall paintings from Phaistos," Journal of Raman Spectroscopy, vol. 43, no. 11, pp. 1663-1670, 2012.

[9] L. Burgio and R. J. H. Clark, "Comparative pigment analysis of six modern Egyptian papyri and an authentic one of the 13th century BC by Raman microscopy other techniques," Journal of Raman Spectroscopy, vol. 31, no. 5, pp. 395-401, 2000.

[10] D. Bersani, P. P. Lottici, F. Vignali, and G. Zanichelli, "A study of medieval illuminated manuscripts by means of portable Raman equipments," Journal of Raman Spectroscopy, vol. 37, no. 10, pp. 1012-1018, 2006.

[11] A. Zoppi, C. Lofrumento, N. F. C. Mendes, and E. M. Castellucci, "Metal oxalates in paints: a Raman investigation on the relative reactivities of different pigments to oxalic acid solutions," Analytical and Bioanalytical Chemistry, vol. 397, no. 2, pp. 841-849, 2010.

[12] R. Aroca, Surface-Enhanced Vibrational Spectroscopy, John Wiley \& Sons, Chichester, UK, 2006.

[13] E. C. Le Ru and P. G. Etchegoin, Principles of Surface-Enhanced Raman Spectroscopy, Elsevier, New York, NY, USA, 2009.

[14] A. C. Albrecht, "On the theory of raman intensities," The Journal of Chemical Physics, vol. 34, no. 5, p. 1476, 1961.

[15] M. Fleischmann, P. J. Hendra, and A. J. McQuillan, "Raman spectra of pyridine adsorbed at a silver electrode," Chemical Physics Letters, vol. 26, no. 2, pp. 163-166, 1974.

[16] P. C. Lee and D. Meisel, "Adsorption and surface-enhanced Raman of dyes on silver and gold sols," Journal of Physical Chemistry, vol. 86, no. 17, pp. 3391-3395, 1982.

[17] E. Vogel, W. Kiefer, V. Deckert, and D. Zeisel, "Laser-deposited silver island films: an investigation of their structure, optical properties and SERS activity," Journal of Raman Spectroscopy, vol. 29, no. 8, pp. 693-702, 1998.

[18] A. V. Whitney, R. P. Van Duyne, and F. Casadio, "Silver island films as substrate for Surface-Enhanced Raman Spectroscopy 
(SERS): a methodological study on their application to Artists' red dyestuffs," in Advanced Environmental, Chemical, and Biological Sensing Technologies III, vol. 5993 of Proceedings of SPIE, Boston, Mass, USA, October 2005.

[19] E. Nalbant Esenturk and A. R. Hight Walker, "Surface-enhanced Raman scattering spectroscopy via gold nanostars," Journal of Raman Spectroscopy, vol. 40, no. 1, pp. 86-91, 2009.

[20] A. Guerrero-Martínez, S. Barbosa, I. Pastoriza-Santos, and L. M. Liz-Marzán, "Nanostars shine bright for you: colloidal synthesis, properties and applications of branched metallic nanoparticles," Current Opinion in Colloid \& Interface Science, vol. 16, no. 2, pp. 118-127, 2011.

[21] J.-Q. Hu, Q. Chen, Z.-X. Xie et al., "A Simple and effective route for the synthesis of crystalline silver nanorods and nanowires," Advanced Functional Materials, vol. 14, no. 2, pp. 183-189, 2004.

[22] S. E. J. Bell and S. J. Spence, "Disposable, stable media for reproducible surface-enhanced Raman spectroscopy," Analyst, vol. 126, no. 1, pp. 1-3, 2001.

[23] M. Leona, J. Stenger, and E. Ferloni, "Application of surfaceenhanced Raman scattering techniques to the ultrasensitive identification of natural dyes in works of art," Journal of Raman Spectroscopy, vol. 37, no. 10, pp. 981-992, 2006.

[24] M. Leona, "Microanalysis of organic pigments and glazes in polychrome works of art by surface-enhanced resonance Raman scattering," Proceedings of the National Academy of Sciences of the United States of America, vol. 106, no. 35, pp. 14757-14762, 2009.

[25] A. Idone, M. Gulmini, A.-I. Henry et al., "Silver colloidal pastes for dye analysis of reference and historical textile fibers using direct, extractionless, non-hydrolysis surface-enhanced Raman spectroscopy," Analyst, vol. 138, no. 20, pp. 5895-5903, 2013.

[26] Z. Jurasekova, E. del Puerto, G. Bruno, J. V. García-Ramos, S. Sanchez-Cortes, and C. Domingo, "Extractionless nonhydrolysis surface-enhanced Raman spectroscopicdetection of historical mordant dyes on textile fibers," Journal of Raman Spectroscopy, vol. 41, no. 11, pp. 1455-1461, 2010.

[27] M. Leona, P. Decuzzi, T. A. Kubic, G. Gates, and J. R. Lombardi, "Nondestructive identification of natural and synthetic organic colorants in works of art by surface enhanced raman scattering," Analytical Chemistry, vol. 83, no. 11, pp. 3990-3993, 2011.

[28] B. Doherty, B. G. Brunetti, A. Sgamellotti, and C. Miliani, "A detachable SERS active cellulose film: a minimally invasive approach to the study of painting lakes," Journal of Raman Spectroscopy, vol. 42, no. 11, pp. 1932-1938, 2011.

[29] B. Doherty, F. Presciutti, A. Sgamellotti, B. G. Brunetti, and C. Miliani, "Monitoring of optimized SERS active gel substrates for painting and paper substrates by unilateral NMR profilometry," Journal of Raman Spectroscopy, vol. 45, no. 11-12, pp. 1153-1159, 2014.

[30] F. Pozzi and M. Leona, "Surface-enhanced raman spectroscopy in art and archaeology," Journal of Raman Spectroscopy, vol. 47, no. 1, pp. 67-77, 2016.

[31] C. Muehlethaler, M. Leona, and J. R. Lombardi, "Review of surface enhanced Raman scattering applications in forensic science," Analytical Chemistry, vol. 88, no. 1, pp. 152-169, 2015.

[32] H. Schweppe, "Indigo and woad," in Artists' Pigments: A Handbook of Their History and Characteristics, R. L. Feller, E. West FitzHugh, and A. Roy, Eds., vol. 3, pp. 81-107, National Gallery of Art, Washington, DC, USA, 1997.

[33] Z. Zhou, G. G. Huang, T. Kato, and Y. Ozaki, "Experimental parameters for the SERS of nitrate ion for label-free semiquantitative detection of proteins and mechanism for proteins to form SERS hot sites: a SERS study," Journal of Raman Spectroscopy, vol. 42, no. 9, pp. 1713-1721, 2011.

[34] D. Stulik, D. Miller, H. Khanjian et al., Solvent Gels for the Cleaning of Works of Art: The Residue Question, edited by V. Dorge, Getty Publications, 2004.

[35] C. Lofrumento, M. Ricci, E. Platania, M. Becucci, and E. Castellucci, "SERS detection of red organic dyes in Ag-agar gel," Journal of Raman Spectroscopy, vol. 44, no. 1, pp. 47-54, 2013.

[36] E. Platania, J. R. Lombardi, M. Leona et al., "Suitability of Agagar gel for the microextraction of organic dyes on different substrates: the case study of wool, silk, printed cotton and a panel painting mock-up," Journal of Raman Spectroscopy, vol. 45, no. 11-12, pp. 1133-1139, 2014.

[37] E. Platania, C. Lofrumento, E. Lottini, E. Azzaro, M. Ricci, and M. Becucci, "Tailored micro-extraction method for Raman/SERS detection of indigoids in ancient textiles," Analytical and Bioanalytical Chemistry, vol. 407, no. 21, pp. 6505-6514, 2015.

[38] C. Lofrumento, E. Platania, M. Ricci, C. Mulana, M. Becucci, and E. M. Castellucci, "The SERS spectra of alizarin and its ionized species: the contribution of the molecular resonance to the spectral enhancement," Journal of Molecular Structure, vol. 1090, pp. 98-106, 2015.

[39] C. Lofrumento, F. Arci, S. Carlesi, M. Ricci, E. Castellucci, and $\mathrm{M}$. Becucci, "Safranin-O dye in the ground state. A study by density functional theory, Raman, SERS and infrared spectroscopy," Spectrochimica Acta Part A: Molecular and Biomolecular Spectroscopy, vol. 137, pp. 677-684, 2015.

[40] S. Gaurav and B. Raja, Digital Color Imaging Handbook, Edited by G. Sharma, CRC Press, New York, NY, USA, 2002.

[41] I. T. Shadi, B. Z. Chowdhry, M. J. Snowden, and R. Withnall, "Semi-quantitative analysis of indigo by surface enhanced resonance Raman spectroscopy (SERRS) using silver colloids," Spectrochimica Acta Part A: Molecular and Biomolecular Spectroscopy, vol. 59, no. 10, pp. 2213-2220, 2003. 

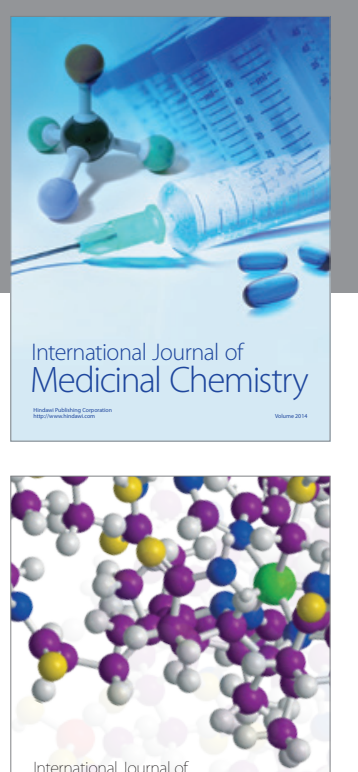

Carbohydrate Chemistry

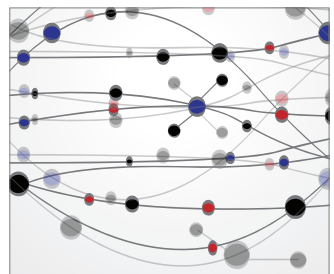

The Scientific World Journal
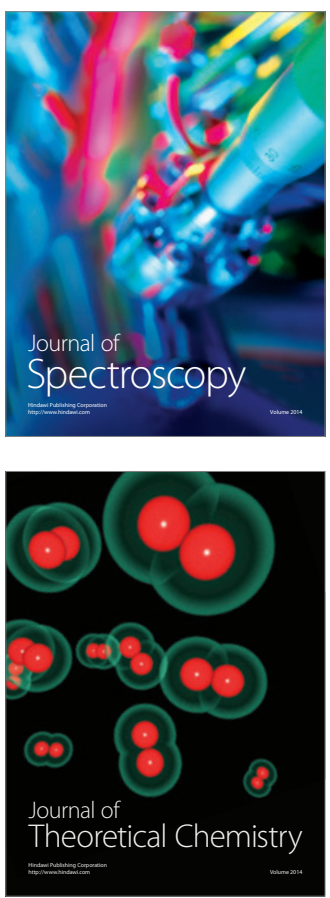
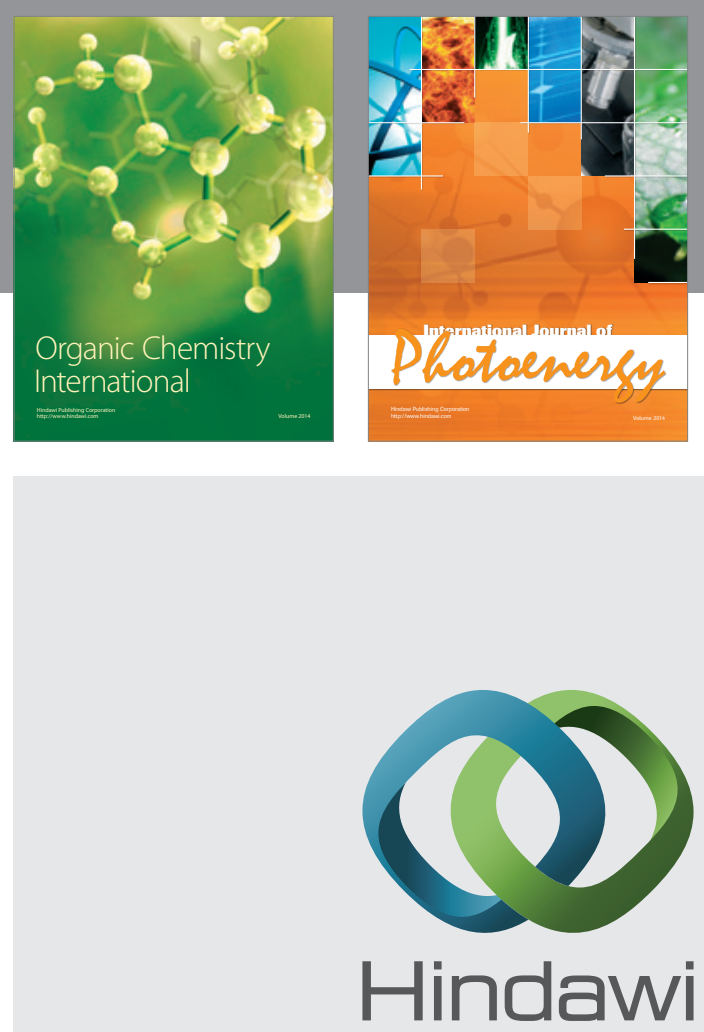

Submit your manuscripts at

http://www.hindawi.com

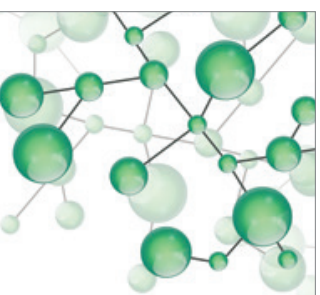

International Journal of

Inorganic Chemistry

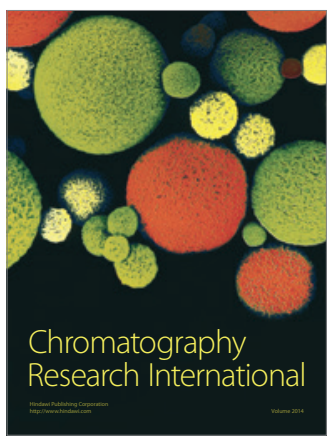

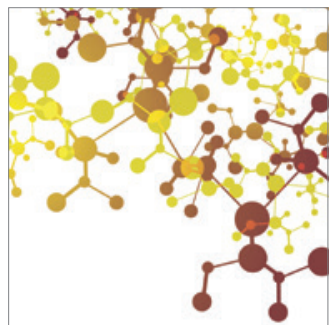

Applied Chemistry
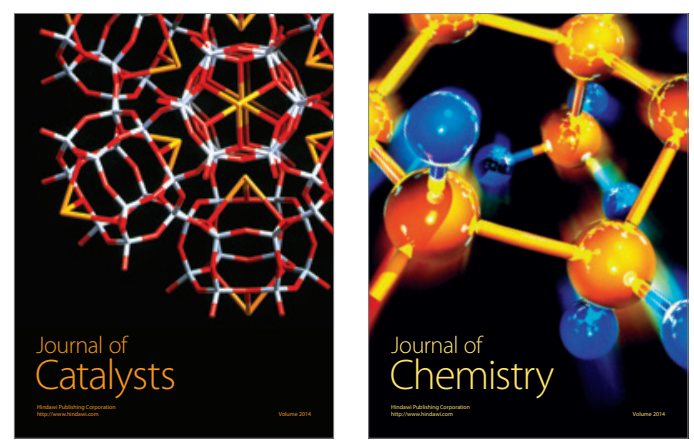
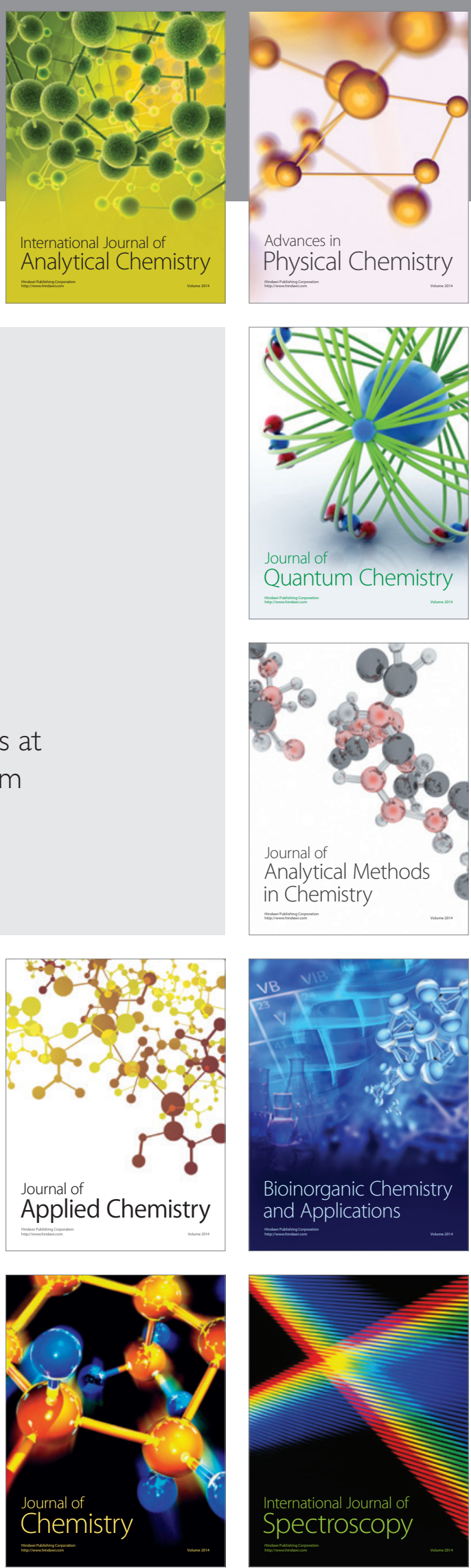